\title{
Computer simulation of the linear and nonlinear optical properties of liquid benzene : its local fields, refractive index and second nonlinear susceptibility
}

Citation for published version (APA):

Janssen, R. H. C., Bomont, J-M., Theodorou, D. N., Raptis, S., \& Papadopoulos, M. G. (1999). Computer simulation of the linear and nonlinear optical properties of liquid benzene : its local fields, refractive index and second nonlinear susceptibility. Journal of Chemical Physics, 110(13), 6463-6474.

https://doi.org/10.1063/1.478549

DOI:

$10.1063 / 1.478549$

Document status and date:

Published: 01/01/1999

Document Version:

Publisher's PDF, also known as Version of Record (includes final page, issue and volume numbers)

Please check the document version of this publication:

- A submitted manuscript is the version of the article upon submission and before peer-review. There can be important differences between the submitted version and the official published version of record. People interested in the research are advised to contact the author for the final version of the publication, or visit the $\mathrm{DOI}$ to the publisher's website.

- The final author version and the galley proof are versions of the publication after peer review.

- The final published version features the final layout of the paper including the volume, issue and page numbers.

Link to publication

\footnotetext{
General rights

- You may freely distribute the URL identifying the publication in the public portal. follow below link for the End User Agreement:

www.tue.nl/taverne

Take down policy

If you believe that this document breaches copyright please contact us at:

openaccess@tue.nl

providing details and we will investigate your claim.
}

Copyright and moral rights for the publications made accessible in the public portal are retained by the authors and/or other copyright owners and it is a condition of accessing publications that users recognise and abide by the legal requirements associated with these rights.

- Users may download and print one copy of any publication from the public portal for the purpose of private study or research.

- You may not further distribute the material or use it for any profit-making activity or commercial gain

If the publication is distributed under the terms of Article 25fa of the Dutch Copyright Act, indicated by the "Taverne" license above, please 


\title{
Computer simulation of the linear and nonlinear optical properties of liquid benzene: Its local fields, refractive index, and second nonlinear susceptibility
}

\author{
R. H. C. Janssen, J.-M. Bomont, and D. N. Theodorou ${ }^{a)}$ \\ Department of Chemical Engineering, University of Patras, GR 26500 Patras, Greece and Institute \\ of Chemical Engineering and High Temperature Chemical Processes, GR 26500 Patras, Greece
}

S. Raptis and M. G. Papadopoulos

Institute of Organic and Pharmaceutical Chemistry, National Hellenic Research Foundation, Vassileos Constantinou 48, GR 11635 Athens, Greece

(Received 25 September 1997; accepted 28 December 1998)

\begin{abstract}
Molecular dynamics (MD) simulation and subsequent analysis of the macroscopic polarization developed in response to "a posteriori" applied electric fields or of spontaneous fluctuations in the instantaneous polarization under zero applied field is used to assess the nonlinear optical properties of a polarizable liquid. Three strategies are proposed for the electrostatic analysis, all using as input static "gas phase" (hyper)polarizabilities, obtained from ab initio calculations. All three strategies are shown to accurately reproduce the experimentally measured refractive index and second nonlinear susceptibility of liquid benzene. The simulation also predicts the distribution of orientations and magnitudes of the local electric fields experienced by the molecules in the liquid, and the nonlinear contributions to the local fields. This approach gives an $8 \%$ higher estimate of the second nonlinear susceptibility of liquid benzene than the Lorentz local field factor approach, in better agreement with experimental values. (C) 1999 American Institute of Physics.

[S0021-9606(99)51612-7]
\end{abstract}

\section{INTRODUCTION}

Nonlinear optical (NLO) processes are becoming increasingly important in the optoelectronics industry. ${ }^{1,2}$ Of great importance are, for instance, high speed all-optical switching and computing, which rely on changes of the refractive index of a material with the intensity of an applied laser field. ${ }^{3}$ Obviously, the efficiency of such a nonlinear process relies heavily on the properties of the materials employed in it. Currently, there is an increasing tendency to study the use of organic molecular and polymeric materials in such applications, since they are easily processable, show high optical damage thresholds, and can in principle be tailored for a specific application by the methods of organic chemistry. ${ }^{1-4}$

Thus far, the study of nonlinear optical properties of molecular materials has mainly focused on identifying molecules showing large nonlinear responses, i.e., molecules having high hyperpolarizabilities. Theoretically, single molecule "gas phase" hyperpolarizabilities have been estimated by $a b$ initio (e.g., Refs. 5 and 6) and semiempirical (e.g., Refs. 7 and 8) quantum chemical approaches. Experimentally, the electric field-induced second-harmonic generation (EFISH) technique ${ }^{9-11}$ and, more recently, the hyperRayleigh scattering (HRS) technique ${ }^{12}$ have been developed and exploited to estimate molecular hyperpolarizabilities in the liquid phase. Gas-phase measurements, ${ }^{13}$ which would

\footnotetext{
a) Author to whom correspondence should be addressed at the University of Patras; electronic mail: doros@ sequoia.chemeng.upatras.gr
}

allow for a direct comparison between experimentally and quantum chemically obtained hyperpolarizabilities, are often not possible, since NLO-active molecules have very high dipole moments, which do not allow evaporating them into the gas phase. Therefore, a local field factor approach, ${ }^{14}$ essentially a direct extension of the classical Lorentz local field approximation for dielectric material properties, ${ }^{15}$ is commonly used to extract single molecule "gas phase" hyperpolarizabilities from experiments performed in the liquid phase. Although the local field factor procedure is generally accepted, there is every reason to doubt its validity in extracting molecular properties such as the (hyper)polarizabilities. ${ }^{15}$ Therefore, in order to compare directly experimental and calculated hyperpolarizabilities, there is a need to establish quantitative relations between the macroscopic material properties measured in experiments and the molecular properties obtained from quantum chemical work. Attempts to accomplish this have been undertaken by Willets and Rice ${ }^{16}$ and Yu and Zerner, ${ }^{17}$ who have tried directly to incorporate the effect of the environment of a molecule into $a b$ initio calculations via a reaction field approach.

We believe that it is inherently more accurate to use a hierarchical scheme which iterates between quantum chemical and statistical mechanical calculations to accomplish the above task in the following way: Gas-phase (hyper)polarizabilities obtained from the $a b$ initio calculations are used as input values in a classical (molecular dynamics or Monte Carlo) simulation, which subsequently produces the distribution of the electric fields experienced by the molecules. 
These fields can, if necessary, be used to correct the gasphase hyperpolarizability values in a new $a b$ initio round, etc. Such a hierarchical approach would be ideally suited to estimating the influence of the environment on the molecular (hyper)polarizabilities (the so-called "solvent effect"). Currently, it is believed that this effect may increase the first hyperpolarizability by a factor of 2 or more relative to the gas phase, with serious consequences on properties. ${ }^{16-19}$

The objective of the work presented in this paper was to establish a quantitative relation between the macroscopic and molecular NLO properties of a molecular liquid and to assess the magnitudes and orientations of the local electric fields experienced by the molecules in such a liquid. Liquid benzene has been chosen as a test case, because of its relative simplicity and the fact that it has been studied extensively both by optical experiments and by simulations.

We have used a combined quantum mechanicalstatistical mechanical approach to accomplish the task: Input to our molecular simulations are static polarizabilities and hyperpolarizabilities obtained from $a b$ initio quantum chemical calculations on isolated molecules. Our calculations show that, for this system, it is not necessary to refine further the $a b$ initio molecular properties of our benzene model. Feedback of our results to the quantum level is not necessary in the current case.

Our approach, implemented using three different strategies, has further allowed us to test the local field factor approach for the nonlinear susceptibility of liquid benzene. We have found that the local field factor approach predicts an $8 \%$ lower second-order nonlinear susceptibility than our approach if we use the same molecular polarizability $\left(\alpha_{i j}\right)$ and hyperpolarizability $\left(\gamma_{i j k l}\right)$ tensors as input. We believe that the difference between the two approaches may well point to inaccuracies in the local field factor approach, because the current approach involves much less simplifying assumptions. Our findings are supported by Stähelin et al., whose experiments on gaseous and liquid acetonitrile also point to an invalidity of the local field factor approach. ${ }^{19}$

Our computational approach utilizes the molecular dynamics technique to generate a sequence of liquid configurations for a fluid consisting of polarizable molecules in the absence of external electric fields. The instantaneous polarization that each configuration would develop when subjected to an electromagnetic field is computed by solving the equations of electrostatics within the configuration of interest, using as input molecular polarizabilities and hyperpolarizabilities calculated from quantum mechanics. Subsequently, the macroscopic polarization of the fluid is obtained by averaging the instantaneous polarization of individual configurations. The coupling of the induced part of the instantaneous polarization to the imposed field is taken into account in weighting configurations during this averaging process. From the computed dependence of the macroscopic polarization on the macroscopic electric field, the linear and nonlinear electric susceptibilities are extracted using two different strategies. As a third strategy, the susceptibilities are extracted from an analysis of spontaneous fluctuations in the instantaneous polarization in an equilibrium liquid free of externally imposed electric fields. All three strategies lead to the same results in the case of benzene.

Several complications have to be overcome in the proposed approach. Generating a trajectory (sequence of configurations of the field-free liquid) requires an accurate knowledge of particle-particle interactions. Not only are these interactions of long-range character (they need to be handled by Ewald summation ${ }^{20-22}$ ), but interparticle forces are also dependent on the local environment of each particle. This latter feature is caused by the polarizable nature of the particles. The interparticle forces that arise due to the polarizability of the particles can in principle be determined selfconsistently (see e.g., Refs. 23 and 24), but this amounts to an enormous increase in computational cost (by more than a factor of 10). In order to keep sufficient computational speed, we resort to a major simplification in the interparticle potential: The sacrifice of the polarizable nature of the molecules during the simulation. Molecular dynamics (MD) trajectories are generated using a force field cast in terms of LennardJones (LJ) interactions and Coulomb interactions between permanent charges only, which, however, reproduces experimentally observed equilibrium structural, thermodynamic, and transport properties satisfactorily. After generating a representative set of liquid configurations with the simplified interparticle potential, the polarizable nature of the molecules is restored, which then allows for calculation of the local electric fields experienced by the molecules, the polarization developing in response to an external field, and the optical properties of the fluid. Our procedure, which is justified in the Sec. II, thus amounts to a decoupling of the electrostatics problem from the generation of the liquid configurations, much in the spirit of the work of Ladanyi et al. ${ }^{25,26}$

The outline of the rest of this paper is as follows. In Sec. II we mention the basic equations governing the macroscopic and microscopic behavior of a NLO material in an externally applied electric field, discuss the molecular model we use, and present our three strategies for extracting the linear and nonlinear susceptibilities. In Sec. III we present and discuss our results on the liquid structure (Sec. III A), the local electric fields (Sec. III B), and the macroscopic optical properties of liquid benzene (Sec. IIIC). Conclusions are drawn in Sec. IV.

\section{THEORETICAL CONSIDERATIONS}

\section{A. Problem formulation and computational strategies employed}

Applying an electric field $E_{0}$ to a macroscopic amount of material generally induces a polarization $P$ in the material, given by ${ }^{27,14}$

$$
\underline{P}=\underline{\underline{\chi}}^{(1)} \cdot \underline{E}+\underline{\underline{\underline{\chi}}}{ }^{(2)}: \underline{E E}+\underline{\underline{\underline{\underline{\chi}}}}^{(3)}: \underline{E E E}+\cdots
$$

The polarization $\underline{P}$ is defined as the material's dipole moment per unit volume. $\underline{E}$ is the macroscopic field in the material, i.e., the field that would be measured if the material were placed in a capacitor. The $E E$ and $\underline{E E E}$ are tensorial terms nonlinear in $\underline{E}$. Note that $\underline{E}$ is not equal to the applied field $E_{0}$, but is given by

$$
\epsilon_{0} \underline{E}_{0}=\epsilon_{0} \underline{E}+\underline{P}_{\text {surf }},
$$


where $\underline{P}_{\text {surf }}$ is the surface polarization of the macroscopic body (see Chaps. 10 and 11 of Ref. 28). The $\underline{\underline{\chi}}^{(1)}, \underline{\underline{\chi}}^{(2)}$, and $\underline{\underline{\underline{\underline{\chi}}}}^{(3)}$ in Eq. (1) are, respectively, the linear susceptibility tensor, and the first and second nonlinear susceptibility tensors. These susceptibilities are the material properties of interest. The $\underline{\underline{\chi}}^{(1)}$ can be related to the refractive index tensor $\underline{\underline{n}}$ of the material via

$$
\underline{\underline{\chi}}^{(1)}=\epsilon_{0}(\underline{n}=\underline{n}=\underline{=})
$$

in which $\epsilon_{0}$ is the dielectric permittivity of vacuum and 1 the unit tensor. Generally, $\underline{\underline{\chi}}^{(1)} \cdot \underline{E} \gg \underline{\underline{\underline{\chi}}}^{(2)}: \underline{E E} \gg \underline{\underline{\underline{\chi}}}^{(3)}: \underline{E E E}{ }^{\bar{c}}$; the nonlinear contributions can only be observed experimentally for $E>10^{5} \mathrm{~V} / \mathrm{m}$. Note that the $\chi^{(n)}$ 's normally are functions of the frequency of the applied field. At optical frequencies, far away from molecular resonances, this frequency dependence may be neglected as a first reasonable approximation. $^{10,15}$

The molecular counterpart of Eq. (1) is given by ${ }^{14}$

$$
\begin{aligned}
& \underline{\mu}_{i}=\underline{\mu}_{0, i}+\underline{\mu}_{i, \mathrm{ind}}=\underline{\mu}_{0, i}+\underline{\alpha} \cdot \underline{E}_{\mathrm{loc}, i}+\underline{\beta}: \underline{E E_{\mathrm{loc}, i}} \\
& +\underline{\underline{\underline{\underline{\gamma}}}} \underline{\underline{E E E_{\mathrm{loc}}, i}}+\cdots
\end{aligned}
$$

in which $E_{\mathrm{loc}, i}$ is the local electric field experienced by mol-

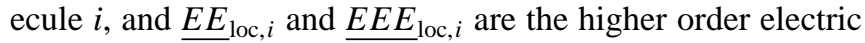
field tensors formed from $\underline{E}_{\text {loc, } i}$, denoting nonlinear contributions to $\underline{\mu}_{i}$. Equation (4) states that the dipole moment of molecule $i$ is determined by a sum of its permanent dipole moment $\underline{\mu}_{0, i}$ (which is zero in the case of benzene), an induction contribution determined by the molecule's polarizability tensor $\alpha$, and contributions nonlinear in $\underline{E}_{\mathrm{loc}, i}$ determined by the hyperpolarizabilities $\beta$ and $\underline{\underline{\underline{y}}}$. The

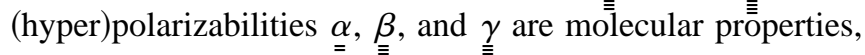
depending on the frequency of the local electric fields. At optical frequencies, far away from electronic resonances, we may again ignore this frequency dependence as a first approximation.

The problem that we are addressing in this paper is how to obtain $\underline{\underline{\chi}}^{(1)}$ and $\chi^{(3)}$ from the molecular (hyper)polarizabilities of benzene $\overline{\bar{b}}$ by electrostatic analysis of a representative set of liquid configurations. $\left(\underline{\underline{x}}^{(2)}\right.$ is zero for isotropic systems.) A second objective is to assess the strength and orientation of $\underline{E}_{\mathrm{loc}, i}$ in the absence of an externally imposed field.

Consider a model molecular fluid of $N$ molecules in volume $V$ at temperature $T$, in which the charge distribution is represented in terms of fixed partial charges, $z_{i a}$, on specific sites $a$ of the molecules $i$ and point dipoles, $\underline{\mu}_{i \text {,ind }}$, at the molecular centers of mass (c.m.). The partial charges are responsible for the permanent dipole moment $\underline{\mu}_{0, i}$ $=\Sigma_{a} z_{i a} \underline{r}_{i a}$ on each molecule, while the induced part of $\underline{\mu}_{i}$ is determined by local fields according to Eq. (4). Following the usual convention of molecular simulations, we will regard the liquid as periodic over length scales exceeding the length of the simulation box. ${ }^{20,22}$ Let

$$
\underline{L}=\left|\begin{array}{ccc}
L_{X} & 0 & 0 \\
0 & L_{Y} & 0 \\
0 & 0 & L_{Z}
\end{array}\right|
$$

be a matrix formed from the edge lengths of the primary simulation box. The contents of the primary box of volume $V$ are copied into image boxes located at positions $L \cdot \underline{m}$ relative to the parent box, $\underline{m}$ being a vector of integer components.

In the presence of a macroscopic field $\underline{E}$, each microscopic configuration $r$ of the fluid will develop an instantaneous polarization $\mathscr{P}$, given by

$\underline{\mathscr{P}}=\mathscr{P}(\underline{r}, \underline{E})=\frac{1}{V} \sum_{i=1}^{N} \underline{\mu}_{i}=\frac{1}{V} \sum_{i=1}^{N}\left(\underline{\mu}_{0, i}+\underline{\mu}_{i, \text { ind }}\right)=\underline{\mathscr{P}}_{0}+\underline{\mathscr{P}}_{\text {ind }}$.

The dipole moments $\underline{\mu}_{i}$ needed for calculating $\mathscr{P}$ are related to the local fields through Eq. (4). In turn, the local field $\underline{E}_{10 c, i}$ at the c.m. of each molecule $i$ may be obtained by iterative self-consistent solution of Eq. (4) together with

$$
\underline{E}_{\mathrm{loc}, i}=-\frac{\partial \phi_{i}}{\partial \underline{r}}+\underline{E}
$$

with the electrostatic potential at $i$ given by

$$
\begin{aligned}
& \phi_{i}=\sum_{\underline{m}=\underline{0}}^{\prime \prime} \sum_{j}^{\prime}\left(\sum_{b} \frac{z_{j b}}{4 \pi \epsilon_{0}\left|\underline{r}_{i, j b}+\underline{L} \cdot \underline{m}\right|}\right.
\end{aligned}
$$

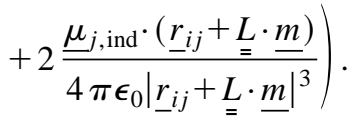

In Eqs. (6) and (7), vector $\underline{r}_{i j}$ points from the c.m. of molecule $j$ to the c.m. of molecule $i$, while vector $\underline{r}_{i, j b}$ points from site $b$ of molecule $j$ to the c.m. of molecule $i$. The prime on the summation over $j$ indicates that $j=i$ should be excluded for $\underline{m}=\underline{0}$. The double prime in the $\underline{m}$ sum denotes that we only sum over the absolutely convergent part of the total sum $\Sigma_{\underline{m}=\underline{0}}$. One has a freedom to construct different shapes of a macroscopic body by summing over $\underline{m}$ in different ways, but, as long as the $\underline{E}$ field in the macroscopic body is homogeneous (which is the case for generalized ellipsoids), the values of the $\underline{E}$ fields differ only by a term $\underline{P}_{\text {surf }} / \epsilon_{0}$, defined in Eq. (2). This $\underline{P}_{\text {surf }}$ is therefore the value of the conditionally convergent part of the total sum $\Sigma_{\underline{m}=\underline{0}}$. Equations (6) and (7), which are formulated in terms of $E$ and $\Sigma_{\underline{m}=\underline{0}}^{\prime \prime}$, are therefore identical to a description in terms of $\underline{E}_{0}$ and the full sum $\Sigma_{\underline{m}=\underline{0}}$. The formulation chosen here avoids the need to explicitly consider $\underline{P}_{\text {surf }}$ in the electrostatics problem, since the absolutely convergent part, $\sum_{\underline{m}=\underline{0}}^{\prime \prime}$, can be obtained directly by Ewald summation ${ }^{29}$ (see Sec. II C).

The macroscopic polarization of the fluid, $\underline{P}$, is given by

$\underline{P}=\frac{\int d \underline{r \mathscr{P}}(\underline{r}, \underline{E}) \exp \left\{-\left[U_{0}(\underline{r})-V \underline{\mathscr{P}}(\underline{r}, \underline{E}) \cdot \underline{E}\right] /\left(k_{B} T\right)\right\}}{\int d \underline{r} \exp \left\{-\left[U_{0}(\underline{r})-V \underline{\mathscr{P}}(\underline{r}, \underline{E}) \cdot \underline{E}\right] /\left(k_{B} T\right)\right\}}$,

where $U_{0}(\underline{r})$ is the potential energy of configuration $r$ in the absence of a macroscopic field $(E=0)$. The second term in the exponential of Eq. (8), involving a coupling of the instantaneous polarization with the field, introduces a bias relative to the probability distribution of configurations in the ab- 
sence of a field and may lead to a field-induced perturbation of the average structure by the field. Denoting by

$$
\langle\mathscr{b}\rangle=\frac{\int d \underline{r} \mathscr{A}(\underline{r}) \exp \left[-U_{0}(\underline{r}) /\left(k_{B} T\right)\right]}{\int d \underline{r} \exp \left[-U_{0}(\underline{r}) /\left(k_{B} T\right)\right]}
$$

the equilibrium ensemble average of a configurationdependent property $\mathscr{C}(\underline{r})$ in the absence of a field, we can rewrite Eq. (8) as

$$
\underline{P}=\frac{\left\langle\underline{P}(\underline{r}, \underline{E}) \exp \left[V \mathscr{P}(r, \underline{E}) \cdot \underline{E} /\left(k_{B} T\right)\right]\right\rangle}{\left\langle\exp \left[V \underline{\mathcal{P}}(\underline{r}, \underline{E}) \cdot \underline{E} /\left(k_{B} T\right)\right]\right\rangle} .
$$

In our work, we are interested in the response to oscillating fields at optical frequencies. Clearly, the rotational degrees of freedom determining the orientation of permanent molecular dipole moments do not have the time to relax in response to the field at these frequencies, i.e., the field $E$ couples only to electronic degrees of freedom and not to the motion of the molecule as a whole. Since molecular orientations are not in equilibrium with respect to the macroscopic field, the permanent dipoles $\underline{\mu}_{i, 0}$, if existent, should not contribute to the weighting factor $\exp \left[V \mathcal{P}(\underline{r}, \underline{E}) \cdot \underline{E} /\left(k_{B} T\right)\right]$, and the correct form of Eq. (10) for our problem is

$$
\underline{P}=\frac{\left\langle\mathscr{P}(\underline{r}, \underline{E}) \exp \left[V \underline{P}_{\text {ind }}(\underline{r}, \underline{E}) \cdot \underline{E} /\left(k_{B} T\right)\right]\right\rangle}{\left\langle\exp \left[V \underline{P}_{\text {ind }}(\underline{r}, \underline{E}) \cdot \underline{E} /\left(k_{B} T\right)\right]\right\rangle} .
$$

Expanding the right-hand side of Eq. (11) in powers of $\underline{E}$ and matching term by term with Eq. (1) leads to the following fluctuation relations for $\underline{\underline{\chi}}^{(1)}$ and $\underline{\underline{\underline{\underline{\chi}}}}^{(3)}$ :

$$
\begin{aligned}
& \underline{\underline{\chi}}^{(1)}=\left\langle\frac{1}{k_{B} T} V \underline{\mathscr{P P}}_{\text {ind }}+\frac{\partial \mathscr{P}_{\text {ind }}}{\partial \underline{E}}\right\rangle, \\
& \underline{\underline{\underline{\underline{\chi}}}}^{(3)}=\left\langle\frac{1}{6} \frac{\partial^{3} \mathscr{P}_{\text {ind }}}{\partial \underline{E}^{3}}+\frac{1}{2 k_{B} T} V \frac{\partial^{2} \mathscr{P}_{\text {ind }}}{\partial \underline{E}^{2}} \underline{\mathscr{P}}_{\text {ind }}\right. \\
& +\frac{\partial \mathscr{P}_{\text {ind }}}{\partial \underline{E}}\left\{\frac{1}{k_{B} T} V \frac{\partial \mathscr{P}_{\text {ind }}}{\partial \underline{E}}+\frac{1}{2}\left(\frac{1}{k_{B} T}\right)^{2} V^{2} \underline{\mathscr{P}}_{\text {ind }} \mathscr{\mathscr { P }}_{\text {ind }}\right\} \\
& +\mathscr{P}\left(\frac{1}{2 k_{B} T} V \frac{\partial^{2} \mathscr{P}_{\text {ind }}}{\partial \underline{E}^{2}}+\left(\frac{1}{k_{B} T}\right)^{2} V^{2} \mathscr{P}_{\text {ind }} \frac{\partial \mathscr{P} \text { ind }}{\partial \underline{E}}\right. \\
& \left.\left.+\frac{1}{6}\left(\frac{1}{k_{B} T}\right)^{3} V^{3} \underline{\mathscr{P}}_{\text {ind }} \mathscr{\mathscr { P }}_{\text {ind }} \underline{\mathscr{P}}_{\text {ind }}\right\}\right) \\
& -\left\langle\frac{1}{k_{B} T} V \underline{\mathscr{P} P}_{\text {ind }}+\frac{\partial \mathscr{P}_{\text {ind }}}{\partial \underline{E}}\right\rangle\left\langle\frac{1}{k_{B} T} V \frac{\partial \mathscr{P}_{\text {ind }}}{\partial \underline{E}}\right. \\
& \left.+\frac{1}{2}\left(\frac{1}{k_{B} T}\right)^{2} V^{2} \underline{\mathscr{P}}_{\text {ind }} \mathscr{P}_{\text {ind }}\right) \text {. }
\end{aligned}
$$

All brackets in Eqs. (12) and (13) denote equilibrium ensemble averages taken under the condition $E=0$, in the sense of Eq. (9). All terms on the right-hand side of Eq. (12) are second-order tensors. All terms on the right-hand side of Eq. (13) are fourth-order tensors.
In the special case of a molecular model with no permanent charges $\left(z_{i a}=0\right)$, Eqs. (5)-(7) applied to the case $E$ $=\underline{0}$ lead to $\mathscr{P}=\mathscr{P}_{\text {ind }}=\underline{0}$ for all configurations and Eqs. (12) and (13) simplify to

$$
\underline{\underline{\chi}}^{(1)}=\left\langle\frac{\partial \mathscr{P}_{\text {ind }}}{\partial \underline{E}}\right\rangle
$$

and

$$
\begin{aligned}
\underline{\underline{\underline{\underline{\chi}}}}^{(3)}= & \left\langle\frac{1}{6} \frac{\partial^{3} \mathscr{P}_{\text {ind }}}{\partial \underline{E}^{3}}\right\rangle+\frac{1}{k_{B} T} V\left[\left\langle\frac{\partial \mathscr{P}_{\text {ind }}}{\partial \underline{E}} \frac{\partial \mathscr{P}_{\text {ind }}}{\partial \underline{E}}\right\rangle\right. \\
& \left.-\left\langle\frac{\partial \mathscr{P}_{\text {ind }}}{\partial \underline{E}}\right\rangle\left\langle\frac{\partial \mathscr{P}_{\text {ind }}}{\partial \underline{E}}\right\rangle\right] .
\end{aligned}
$$

Equation (15) shows that $\chi^{(3)}$ is shaped by two equilibrium, field-free contributions: ${ }^{\prime}$ The average of a "purely third-order" term, and the variance of a "first-order" term whose average equals $\underline{\underline{\chi}}^{(1)}$. In this respect, Eq. (15) is reminiscent of Eq. (4) of Ref. 30 for the Kerr constant describing electric field-induced birefringence in a system of axially symmetric molecules.

We use the formulation presented above to compute $\underline{\underline{\chi}}^{(1)}$ and $\underline{\underline{\underline{\chi}}}^{(3)}$ by three different strategies. Our first $(\underline{P}(\underline{E})$-fit strategy considers a number of fields $E$ of different magnitudes being applied on the macroscopic fluid. For each field $\underline{E}$, the macroscopic polarization $\underline{P}$ is calculated via Eq. (11) as a ratio of two equilibrium ensemble averages over configurations sampled in the course of a simulation of the fieldfree fluid. The macroscopic fields $E$ are low enough, such that the weighting factor $\exp \left[V \mathcal{P}_{\text {ind }}(\underline{r}, \underline{E}) \cdot \underline{E} /\left(k_{B} T\right)\right]$ is not too different from 1 and most of the configurations visited in the field-free simulation contribute significantly to the field-on averages, i.e., the sampling for computing $\underline{P}$ through Eq. (11) is efficient. Once $P$ has been obtained for the different imposed values of $E$, a nonlinear regression based on Eq. (1) with $\chi^{(2)}=0$ is performed on all $(\underline{E}, \underline{P})$ pairs to extract $\underline{\underline{\chi}}^{(1)}$ and $\underline{\underline{\underline{\chi}}}^{(3)}$. The quality of the fit is used as a guide to ensure that the imposed $\underline{E}$ values are weak enough for higher than third-order terms in Eq. (1) to be unimportant.

Our second (subtraction) strategy is again based on computing $P(E)$, but utilizes only a single value for the magnitude of the imposed field $\underline{E}$. For that $E, P$ is calculated in two ways: First, Eq. (11) is applied as in the $\underline{P}(\underline{E})$ - fit strategy, using full values for the molecular properties $\underline{\underline{\alpha}}$ and $\underline{\underline{\underline{\gamma}}}$. Then, the calculation is performed for a second time using the full $\underline{\alpha}$, but setting $\underline{\underline{\underline{\gamma}}}$ equal to zero. The response com-

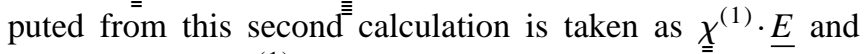
used to extract $\underline{\underline{\chi}}^{(1)}$. On the other hand, the difference between the first and second responses is taken as $\chi^{(3)} \vdots \underline{E E E}$ [compare Eq. (1)] and used to compute $\chi^{(3)}$. Clearly, this is an approximate calculation that should give the same results as the $P(E)$-fit strategy only for weakly nonlinear substances. The incentive for implementing it here is that, through its consideration of a single field magnitude, it is much less expensive computationally than the $\underline{P}(\underline{E})$ fit.

Our third (fluctuation) strategy is based directly on the fluctuation equations (12) and (13). $\underline{\underline{\chi}}^{(1)}$ and $\chi^{(3)}$ are computed as ensemble averages over the sampled field-free con- 
figurations. For each configuration, the required derivatives of $\mathcal{P}_{\text {ind }}$ with respect to $E$ are obtained numerically by finite differences, by imposing on the configuration a small-stepsize grid of $\underline{E}$ values around $\underline{E}=\underline{0}$ and solving the electrostatics problem at each value. The fluctuation strategy is the most rigorous, but also the most demanding computationally of the three, owing to the finite difference derivative calculations it entails.

Details of the numerical implementation of the three strategies are given in Sec. II C.

To generate representative samples of equilibrium fieldfree configurations, one can use either Monte Carlo (MC) or molecular dynamics (MD) methods. ${ }^{20,22}$ We have chosen to employ MD. Strictly, the interaction potential $U_{0}(r)$ used to generate MD trajectories should incorporate partial chargeinduced dipole and induced dipole-induced dipole interactions computed from the $z_{i a}$ and instantaneous $\underline{\mu}_{i \text {,ind }}$ values through self-consistent solution of Eqs. (4), (6), and (7) with $\underline{E}=\underline{0}$ at every simulation step. In practice, such a MD integration scheme would be exceedingly time consuming. Furthermore, reliable intermolecular force fields incorporating polarization effects are unavailable. Instead, we have generated our MD trajectories using a conventional force field proposed in the literature, which is cast in terms of pairwise LJ interactions between atoms and Coulomb interactions between partial charges (see Sec. II C). In other words, our MD trajectories are generated with a potential energy function of the form

$$
\begin{aligned}
U= & \sum_{i} \sum_{a} \sum_{j \neq i} \sum_{b}\left(\frac{A_{a b}}{r_{i a, j b}^{12}}-\frac{B_{a b}}{r_{i a, j b}^{6}}\right) \\
& +\sum_{\underline{m}=\underline{0}} \sum_{i} \sum_{a} \sum_{j}^{\prime}\left(\sum_{b} \frac{z_{i a} z_{j b}}{4 \pi \epsilon_{0}\left|\underline{r}_{i a, j b}+\underline{L} \cdot \underline{m}\right|}\right) .
\end{aligned}
$$

The symbol $\underline{r}_{i a, j b}$ denotes the vector pointing from atom $b$ of molecule $j$ to atom $a$ of molecule $i$. Coulomb forces were summed with the Ewald method.

For each molecular configuration stored in the course of the MD trajectory, we solve the full electrostatics problem using Eqs. (4), (6), and (7) under zero or nonzero imposed $\underline{E}$ to determine $E_{\text {loc, } i}, \mathscr{P}$, and $\mathscr{P}_{\text {ind }}$, hence $P$ via Eq. (11). Decoupling the electrostatics problem from the problem of generating MD trajectories affords a manageable scheme for the computational prediction of macroscopic susceptibilities.

\section{B. Molecular model and simulation details}

A 12-site molecular model is used for benzene. ${ }^{31-37}$ The LJ parameters we employ are taken from the work of Bartell et al. ${ }^{35}$ The $\mathrm{CC}$ and $\mathrm{CH}$ bond lengths, respectively, 1.401 and $1.031 \AA$, are also taken from Ref. 35. Partial charges of $z=0.147|e|$ were located at the $\mathrm{C}$ and $\mathrm{H}$ atoms. The value of the charges was chosen to obtain reasonable agreement with the range of experimentally observed quadrupole moments ${ }^{37}$ and with the partial charges obtained from $a b$ initio quantum calculations. $^{38}$

In the electrostatics calculations, assigning a pointdipole moment to the c.m. of each molecule was considered to be sufficient to mimic the molecular response to the local

\begin{tabular}{|c|c|}
\hline CC bond length $(\AA)$ & 1.401 \\
\hline $\mathrm{CH}$ bond length $(\AA)$ & 1.031 \\
\hline Partial H charge $(e)$ & 0.147 \\
\hline Partial C charge $(e)$ & -0.147 \\
\hline \multicolumn{2}{|l|}{ LJ parameters } \\
\hline$A_{\mathrm{CC}}\left(\mathrm{kcal} \mathrm{mol}^{-1} \AA^{12}\right)$ & 692949.3 \\
\hline$B_{\mathrm{CC}}\left(\mathrm{kcal} \mathrm{mol}^{-1} \AA^{6}\right)$ & 547.6 \\
\hline$A_{\mathrm{CH}}\left(\mathrm{kcal} \mathrm{mol}^{-1} \AA^{12}\right)$ & 88214.6 \\
\hline$B_{\mathrm{CH}}\left(\mathrm{kcal} \mathrm{mol}^{-1} \AA^{6}\right)$ & 103.7 \\
\hline$A_{\mathrm{HH}}\left(\mathrm{kcal} \mathrm{mol}^{-1} \AA^{12}\right)$ & 5021.5 \\
\hline$B_{\mathrm{HH}}\left(\mathrm{kcal} \mathrm{mol}^{-1} \AA^{6}\right)$ & 15.82 \\
\hline \multicolumn{2}{|l|}{ Polarizability $\alpha$ (a.u. $)^{\mathrm{a}}$} \\
\hline$\alpha_{x x}=\alpha_{y y} \quad=$ & 80.03 \\
\hline$\alpha_{z z}$ & 44.74 \\
\hline \multicolumn{2}{|l|}{ Second hyperpolarizability $\underline{\gamma}{\text { (a.u. })^{\mathrm{a}}}$} \\
\hline$\gamma_{x x x x}=\gamma_{y y y y}$ & 2421.5 \\
\hline$\gamma_{z z z z}$ & 2176.5 \\
\hline$\gamma_{x x y y}=\gamma_{x y x y}=\gamma_{x y y x}=\gamma_{y y x x}=\gamma_{y x y x}=\gamma_{y x x y}$ & 825 \\
\hline$\gamma_{x x z z}=\gamma_{x z x z}=\gamma_{x z z x}=\gamma_{z z x x}=\gamma_{z x z x}=\gamma_{z x x z}$ & \\
\hline$=\gamma_{y y z z}=\gamma_{y z y z}=\gamma_{y z z y}=\gamma_{z z y y}=\gamma_{z y z y}=\gamma_{z y y z}$ & 995.5 \\
\hline
\end{tabular}

TABLE I. Molecular data employed in MD simulation.

$\mathrm{a}_{z}$ axis is chosen perpendicular to the plane of the benzene-ring.

environment; calculations on crystalline benzene, in which a point dipole was assigned to each $\mathrm{CH}$ group of the molecule, have given nearly identical results for the refractive index tensor as a single molecular dipole calculation (see Ref. 29 and references cited therein).

For the computation of the polarizability $(\underset{=}{\alpha})$ and second hyperpolarizability $(\underline{\underline{\gamma}})$ components of the benzene molecule we employed field-dê⿳亠丷厂彡⿱ pendent energies, $U(E)$, determined at the MP4[SDQ] level of theory, ${ }^{39,40}$ by using the GAUSSIAN 94 program. ${ }^{41}$ The basis set reported by Sadlej has been used. ${ }^{42}$ The field-dependent energies, $U(E)$, the electric field strengths, $E$, varied between \pm 0.002 and \pm 0.008 a.u. in steps of 0.001 a.u., and the property components are connected by a set of linear equations which are solved by a singular value decomposition method (SVD). ${ }^{43}$ The molecular geometry used in the quantum calculations is as described in Ref. 44. It differs slightly from the geometry used in the MD simulation. The reported (hyper)polarizability values are static, no frequency dependence is included. Note that the first hyperpolarizability $\underset{\equiv}{\beta}$ is zero for the centrosymmetric benzene molecule. ${ }^{1}$ All $\stackrel{\equiv}{\equiv}$ olecular data employed in the MD simulations are summarized in Table I. In this table, the values of the (hyper)polarizability tensor elements are given in atomic units, but conversion to SI units is straightforward ${ }^{45}$ (1 a.u. of $\alpha=0.164867 \times 10^{-40} \mathrm{C}^{2} \mathrm{~m}^{2} \mathrm{~J}^{-1}$ and 1 a.u. of $\gamma$ $=0.62359 \overline{\overline{7}} \times 10^{-64} \mathrm{C}^{4} \mathrm{~m}^{4} \mathrm{~J}^{-3}$ ). Elements of the (hyper)polarizability tensor not mentioned in Table I are zero due to molecular symmetries.

The calculated (hyper)polarizability tensor elements lead to orientationally averaged (hyper)polarizabilities of, respectively, $\alpha=1 / 3 \Sigma_{i} \alpha_{i i}=68.27$ a.u. and $\gamma=\frac{1}{15} \Sigma_{i} \Sigma_{j}\left(\gamma_{i i j j}+\gamma_{i j j i}\right.$ $\left.+\gamma_{i j i j}\right)=2530$ a.u.. These compare reasonably well to the experimental values of $\alpha=69.51$ a.u. $^{46}$ and $\gamma=2727$ a.u., ${ }^{47}$ as determined from gas-phase measurements (Ref. 48 reports a value of $\gamma=4090$ a.u., but this value should be multiplied 
by a factor $2 / 3$ to allow comparison to quantum calculations; see Appendix D of Ref. 10).

Our MD simulation was performed on a system of $N$ $=72$ molecules. The start configuration for the simulations was the crystal structure constructed from data of Craven et l. $^{48}$ Initial velocities were assigned to the atoms according to a Maxwell-Boltzmann distribution. Results from one run, about 270 ps long, are presented. We did not save data during the equilibration part of the runs. The 270 ps are fully equilibrated and completely used for data collection. Molecular positions and velocities were saved every $0.1 \mathrm{ps}$.

The simulation was run in a box of $22.5 \times 21.0 \times 22.5 \AA$ with periodic boundary conditions at average temperature and pressure of $300 \mathrm{~K}$ and 200 bar. A cutoff distance of $10 \AA$ was used for the LJ interactions.

The intramolecular constraint forces were handled by the constraint dynamics method of Edberg, Evans, and Morriss. ${ }^{49}$ During the simulation, three primary atoms per molecule, determining the orientation and position of the molecular plane, were tracked. The positions of the other atoms were determined at each time step from the positions of the primary atoms. ${ }^{50}$ The equations of motion were integrated via a fourth-order predictor-corrector scheme ${ }^{20}$ with a time step of 1 fs. Excellent energy conservation was observed for the MD run (maximum and minimum energies obtained during the run differed by less than $0.1 \%$ ).

\section{Electrostatics}

The electrostatics problem is described by Eqs. (4), (6), and (7). The equations have to be solved for each fluid configuration saved during the MD run. In order to do so we have used the Ewald summation technique. ${ }^{20-22}$ Although the periodicity assumed for the model liquid when using this technique is clearly artificial, we believe that the method provides a more satisfactory representation of the real situation than a simple cutoff for the electrostatic forces, as practiced in for instance Ref. 23.

Utilizing the above-mentioned approach we have rewritten Eqs. (6) and (7) as

$$
\underline{E}_{\mathrm{loc}, i}=\underline{E}+\underline{E}_{\mathrm{PC}, i}+\underline{E}_{\mathrm{dip}, i},
$$

with the electric field that is due to the partial charges $b$ of molecules $j$ given by

$$
\begin{aligned}
4 \pi \epsilon_{o} \underline{E}_{\mathrm{PC}, i}= & \sum_{j \neq i}^{N} \sum_{b} z_{j b}\left(\frac{\operatorname{erfc}\left(\kappa r_{i, j b}\right)}{r_{i, j b}^{3}}\right. \\
& \left.+2 \frac{\kappa}{\sqrt{\pi}} \frac{\exp \left(-\kappa^{2} r_{i, j b}^{2}\right)}{r_{i, j b}^{2}}\right) \underline{r}_{i, j b} \\
& -\frac{1}{V} \sum_{\underline{k} \neq \underline{0}} \sum_{j=1}^{N} \sum_{b} \frac{4 \pi z_{j b}}{k^{2}} \exp \left(\underline{i k} \cdot \underline{r}_{i, j b}\right) \\
& \times \exp \left(-k^{2} / 4 \kappa^{2}\right) i \underline{k}
\end{aligned}
$$

and the part of the field that is due to the induced dipoles of the molecules $j \neq i$, given by

$$
\begin{aligned}
4 \pi \epsilon_{0} \underline{E}_{\mathrm{dip}, i}= & -\sum_{j \neq i}^{N}\left(\frac{\operatorname{erfc}\left(\kappa r_{i j}\right)}{r_{i j}^{3}}+2 \frac{\kappa}{\sqrt{\pi}} \frac{\exp \left(-\kappa^{2} r_{i j}^{2}\right)}{r_{i j}^{2}}\right) \underline{\mu}_{j} \\
& +\left(\underline{r}_{i j} \cdot \underline{\mu}_{j}\right) \sum_{j \neq i}^{N}\left(3 \frac{\operatorname{erfc}\left(\kappa r_{i j}\right)}{r_{i j}^{5}}\right. \\
& \left.+2 \frac{\kappa}{\sqrt{\pi}} \frac{\exp \left(-\kappa^{2} r_{i j}^{2}\right)}{r_{i j}^{2}}\left(3 / r_{i j}^{2}+2 \kappa^{2}\right)\right) \underline{r}_{i j} \\
& +-\frac{1}{V} \sum_{\underline{k} \neq \underline{0}} \sum_{j=1}^{N} \frac{4 \pi \underline{\mu_{j}} \cdot \underline{k}}{k^{2}} \exp \left(\underline{i} \cdot \underline{r}_{i j}\right) \\
& \times \exp \left(-k^{2} / 4 \kappa^{2}\right) \underline{k}+\frac{4}{3} \frac{\kappa^{3}}{\sqrt{\pi}^{\underline{\mu}}} .
\end{aligned}
$$

Note that $\underline{E}_{\mathrm{PC}, i}$ corresponds to the first term in Eq. (7) and $\underline{E}_{\text {dip }, i}$ corresponds to the second term in Eq. (7). In Eqs. (18) and (19), erfc denotes the complementary error function, defined by $\operatorname{erfc}(x)=1-2 / \sqrt{\pi} \int_{0}^{x} e^{-t^{2}} d t$. Equations (18) and (19) are derived by straightforward extension of the derivation of the potential energy expressions Eqs. (5.20) and (5.21) of Ref. 20, using the ideas outlined in Appendix B of Ref. 22. In these equations, the parameter $\kappa$, which determines the width of the Gaussian screening distribution, has to be chosen large enough to effectively screen the partial charges (i.e., to ensure rapid convergence of the real space sums in the equations such that only screened electrostatic interactions within the parent box have to be considered), but small enough to limit the number of wave vectors $k$ that need to be taken into account to let the Fourier space sums converge. Convergence tests have let us choose $\kappa$ $=6 \sqrt{3} / \sqrt{L_{X}^{2}+L_{Y}^{2}+L_{Z}^{2}} \quad$ and $\quad|\underline{k}|=2 \pi \mid\left(m_{X} / L_{X}, m_{Y} / L_{Y}\right.$, $\left.m_{Z} / L_{Z}\right) \mid$ with each of the $m_{X}, m_{Y}, m_{Z}$ assuming integer values between -6 and 6 as the terms in the $k$-space sum. This choice proved more than sufficient to ensure convergence. In the Fourier sum of Eqs. (18) and (19) we do not include the $\underline{k}=\underline{0}$ term, since it corresponds to the conditionally convergent part of the PC and dipolar fields, ${ }^{22,29}$ i.e., the value of the $\underline{k}=\underline{0}$ term is given by $\underline{P}_{\text {surf }}$.

Substitution of Eqs. (4), (18), and (19) in Eq. (17) gives a set of $72 \times 3$ coupled equations for the $x, y$, and $z$ components of $\underline{E}_{\text {loc, } i}$. This has been solved iteratively via a standard Newton-Raphson scheme. Note that ignoring the nonlinear terms in Eq. (4) linearizes the electrostatics problem. It was checked that our solution procedure converged in one iteration for this case.

Setting $\underline{E}=\underline{0}$ in Eq. (17) allows one to obtain the distribution of local electric fields experienced by the molecules in the material in the absence of an external field. We have investigated both the linear case, in which the hyperpolarizability contribution to the induced dipole moment is ignored, and the full problem including nonlinear terms in Eq. (4). Explicit nonlinear contributions to the local fields were obtained by subtraction of the linear from the full solution. 
To obtain the optical material properties, the electrostatics equations have been solved with $\underline{E}_{\mathrm{PC}, i}=\underline{0}$ for both zero and nonzero values of $\underline{E}$ (see Sec. II A). Setting $\underline{E}_{\mathrm{PC}, i}=0$ is common practice $^{29}$ in nonlinear optics work on crystals and serves to separate the optical contributions to the fluid polarization $P$ from the frequency-independent PC contributions. ( $\underline{E}_{\mathrm{PC}, i} \underline{E}_{\mathrm{PC}, i} \underline{E}$ contributions to $\underline{P}$ have a different frequency dependence than the desired $E E E$ contribution, which is observed in actual NLO experiments.)

Reweighting by the factor $\exp \left[V \mathscr{P}_{\text {ind }} \cdot \underline{E} /\left(k_{B} T\right)\right]$ in Eq. (11) is only practical if $V \mathscr{P}_{\text {ind }} \cdot \underline{E} /\left(k_{B} T\right)=\bar{\Sigma}_{i} \underline{\mu}_{i \text {,ind }} \cdot \underline{E} /\left(k_{B} T\right)$ $\leqslant 1$. In the case of benzene at $300 \mathrm{~K}$ this condition is obeyed for $E$ values well over $10^{8} \mathrm{~V} / \mathrm{m}$, i.e., beyond the field intensities used in experimental setups. For larger $E$ 's, reweighting the field-free configurations would give poor results, because in this case the relative importance of a few configurations would overshadow all the others.

In implementing the $P(E)$-fit strategy for the calculation of $\chi^{(1)}$ and $\chi^{(3)}$ we have applied fields $\underline{E}$ of magnitude $10^{6}$, $5 \times 10^{6}, 10^{7}, 5 \times 10^{7}, 10^{8}$, and $5 \times 10^{8} \mathrm{~V} / \mathrm{m}$ along each coordinate direction. The subtraction strategy was implemented using an $\underline{E}$ field of $10^{7} \mathrm{~V} / \mathrm{m}$. For the fluctuation strategy, derivatives of the components of the instantaneous induced polarization with respect to the components of the field, required in Eqs. (12) and (13), were estimated numerically by the finite difference schemes:

$$
\begin{aligned}
& \left.\frac{\partial \mathscr{P}_{\text {ind }}}{\partial E}\right|_{\underline{E}=\underline{0}}=\frac{\mathscr{P}_{\text {ind }}(\Delta E)-\mathscr{P}_{\text {ind }}(-\Delta E)}{2 \Delta E}+\mathscr{Q}\left(\Delta E^{2}\right), \\
& \left.\frac{\partial^{2} \mathscr{P}_{\text {ind }}}{\partial E^{2}}\right|_{\underline{E}=\underline{0}}=\frac{\mathscr{P}_{\text {ind }}(\Delta E)-2 \mathscr{P}_{\text {ind }}(0)+\mathscr{P}_{\text {ind }}(-\Delta E)}{\Delta E^{2}}+\mathscr{O}\left(\Delta E^{2}\right), \\
& \left.\frac{\partial^{3} \mathscr{P}_{\text {ind }}}{\partial E^{3}}\right|_{\underline{E}=\underline{0}}=\frac{\mathscr{P}_{\text {ind }}(2 \Delta E)-2 \mathscr{P}_{\text {ind }}(\Delta E)+2 \mathscr{P}_{\text {ind }}(-\Delta E)-\mathscr{P}_{\text {ind }}(-2 \Delta E)}{2 \Delta E^{3}}+\mathscr{O}\left(\Delta E^{2}\right) .
\end{aligned}
$$

A step size of $\Delta E=10^{7} \mathrm{~V} / \mathrm{m}$ was used in all finite difference calculations, after confirming that all estimates obtained are independent of the exact step size used in this range of $\Delta E$.

From the estimated $\underline{\underline{\chi}}^{(1)}$, values of the refractive index tensor $\underset{=}{n}$ were obtained via Eq. (3) for all three strategies employed.

\section{RESULTS AND DISCUSSION}

This section is divided in three parts. First, the equilibrium fluid structure is compared to experimental $\mathrm{X}$-ray diffraction data (Sec. III A); secondly, results for the local fields experienced by the molecules in the model liquid are shown (Sec. III B); finally, we present results for the macroscopic optical properties of the model fluid in Sec. III C. These results are also compared to experimental measurements.

\section{A. Liquid structure}

In Fig. 1 the Fourier-transform, $\hat{h}_{\mathrm{CC}}(k)$, of the CC-radial distribution function is compared to the $\hat{h}_{\mathrm{CC}}(k)$ obtained from x-ray diffraction data by Narten. ${ }^{51}$ The $\hat{h}_{\mathrm{CC}}(k)$ is defined by

$$
\hat{h}_{\mathrm{CC}}(k)=4 \pi \rho \int_{0}^{\infty}\left(g_{C C}(r)-1\right) \frac{\sin k r}{k r} r^{2} d r,
$$

where $\rho$ denotes the liquid density in molecules $/ \AA^{3}$ and $g_{\text {CC }}(r)$ is the CC-pair distribution function. It is seen from Fig. 1 that there is reasonable agreement between the liquid structures seen in experiment and theory, despite the fact that the simulation is run at $T=295 \mathrm{~K}$ and $p=200$ bar, whereas
$T_{\text {exptl }}=298 \mathrm{~K}$ and $p_{\text {exptl }}=1$ atm. (The agreement obtained illustrates the general fact that liquid structure is not a very sensitive function of pressure.)

In our opinion, Fig. 1 offers strong support for the utility of the benzene model that was employed in the MD simulation. Further support comes from the molecular selfdiffusivity, estimated from the $\mathrm{MD}$ as $D_{s}=(1.7 \pm 0.1)$ $\times 10^{-5} \mathrm{~cm}^{2} / \mathrm{s}$, in reasonable agreement with the range of experimental values that have been reported. ${ }^{32}$ Furthermore,

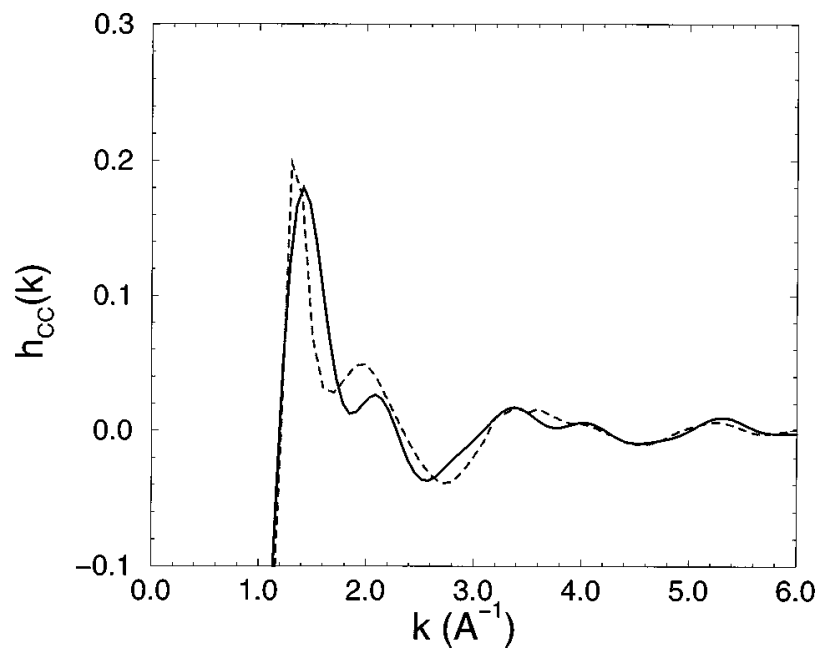

FIG. 1. Comparison of predicted and experimental x-ray diffraction data for liquid benzene. The experimental data (dashed line) at $T=298 \mathrm{~K}$ and $p$ $=1 \mathrm{~atm}$ are taken from Ref. 51. The full line is calculated from the simulation data $(T=295 \mathrm{~K}, p=200 \mathrm{bar})$. 


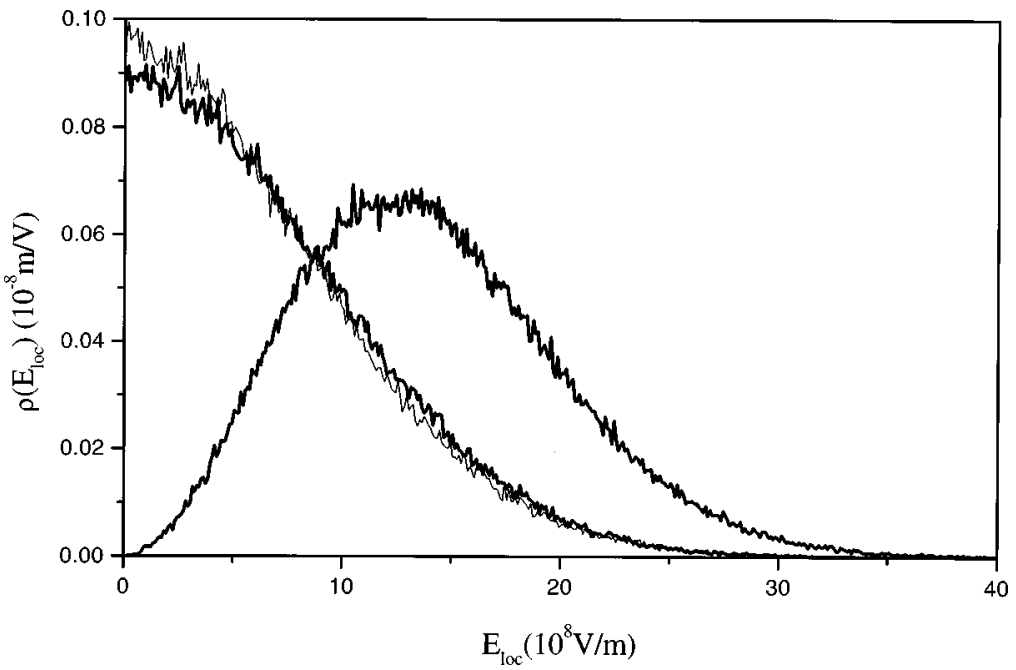

FIG. 2. Normalized distribution of electric field strengths experienced by the molecular c.m.. The curve displaying a maximum represents the distribution of magnitudes of the local field vector, while the other two curves depict the distributions of the magnitudes of local field components along the molecular $z$ axis (the thin line) and $x$ axis (the thick line). The distribution along the $y$ axis coincides with that along the $x$ axis, and is omitted for clarity. we have noticed that the molecular model used by us produced structural results remarkably similar to the model employed by Linse et al. ${ }^{31-33}$ All in all, we believe that our MD simulation of the benzene liquid is as good as any other simulation employing a 12-site model. Further structural, dynamic, and thermodynamic data are not shown, but may be obtained from us.

\section{B. Local fields}

The main results obtained for the local fields $\underline{E}_{\mathrm{loc}, i}$ experienced by the molecules in the absence of an external field are shown in Figs. 2-4. These results were obtained by solving Eqs. (4), (6)-(7) [equivalently, Eqs. (4), (17)-(19)] with $\underline{E}=\underline{0}$ for each liquid configuration. Note that, since $\underline{E}=\underline{0}$, no reweighting of configurations in Eq. (11) was necessary.

Figure 2 depicts the distribution of local field strengths monitored along the molecular $x$ and $z$ axes. (The distribution monitored along the $y$ axis is identical to that measured along the $x$ axis.) We also show the distribution of the magnitude of the local field strength in Fig. 2 (the magnitude is given by $\left.E_{\mathrm{loc}}=\sqrt{E_{\mathrm{loc}, x}^{2}+E_{\mathrm{loc}, y}^{2}+E_{\mathrm{loc}, z}^{2}}\right)$. Note that the magnitudes are skewed toward large $E$ with a peak-value at $E_{\text {loc }}$
$=1.3 \times 10^{9} \mathrm{~V} / \mathrm{m}$. A reaction field analysis, presented in Chap. 3 of Ref. 15 , estimated a value of $4.2 \times 10^{9} \mathrm{~V} / \mathrm{m}$ for liquid nitrobenzene (for which the fields are expected to be higher due to the dipolar nature of the molecule). Note also from Fig. 2 that we have found slight differences between the distribution monitored in the $z$ direction, i.e., perpendicular to the ring, and in the $x$ and $y$ directions. The local fields in the $z$ direction tend to be slightly lower than in the $x$ or $y$ direction, which can be explained by the lower polarizability, $\alpha_{z z}$, of a benzene molecule along the molecular $z$ axis (see Table I): Since the molecular polarizability is lower perpendicular to the ring, its induced dipole moment in the $z$ direction is smaller, resulting in a lower reaction field $E_{\mathrm{loc}, z}$ perpendicular to the ring.

In Fig. 3 explicit nonlinear contributions have been isolated. The distributions of nonlinear contributions monitored along the molecular $x$ and $z$ axes are shown. The most prominent feature observed when comparing Figs. 2 and 3 is the very small magnitude of the nonlinear contribution $\left[\mathcal{O}\left(10^{5}-10^{6} \mathrm{~V} / \mathrm{m}\right)\right]$ relative to the total field strength $\left[\mathcal{O}\left(10^{9}\right.\right.$ $\mathrm{V} / \mathrm{m})$ ], i.e., less than $0.1 \%$. As could have been anticipated

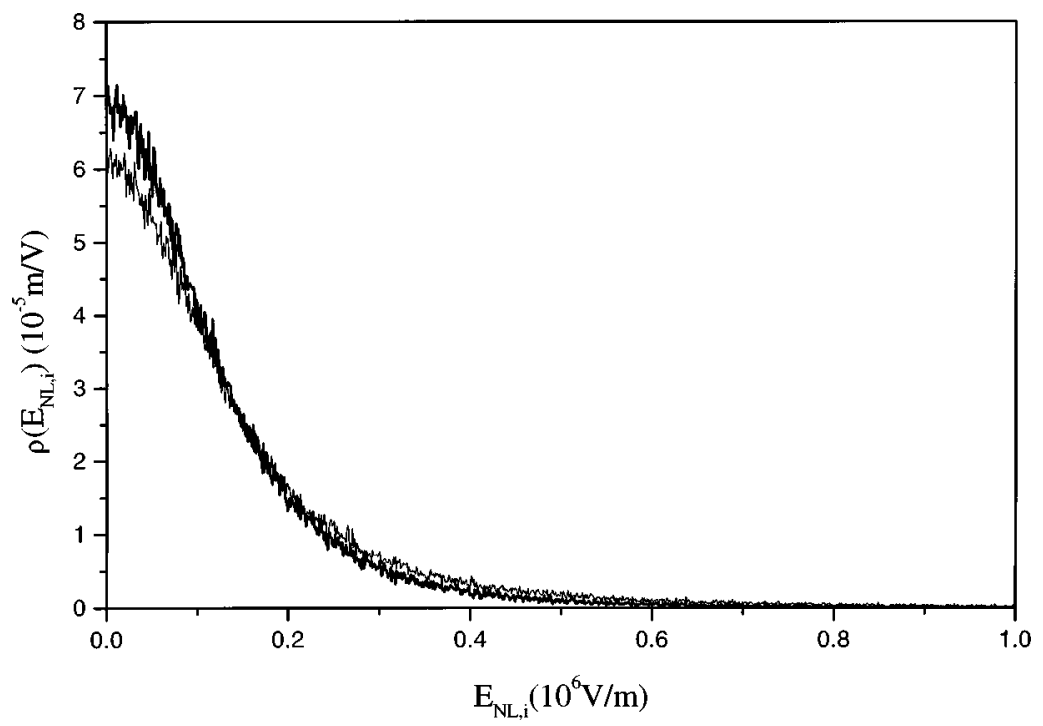

FIG. 3. Normalized distribution of the explicit nonlinear contributions to the magnitudes of the components of the local fields experienced by the c.m. of the molecules. The thin line represents the nonlinear contributions monitored along the molecular $z$ axis. The thick line is obtained by monitoring along the $x$ axis. The distribution monitored along the molecular $y$ axis coincides with that monitored along the $x$ axis, and is not shown for clarity. 


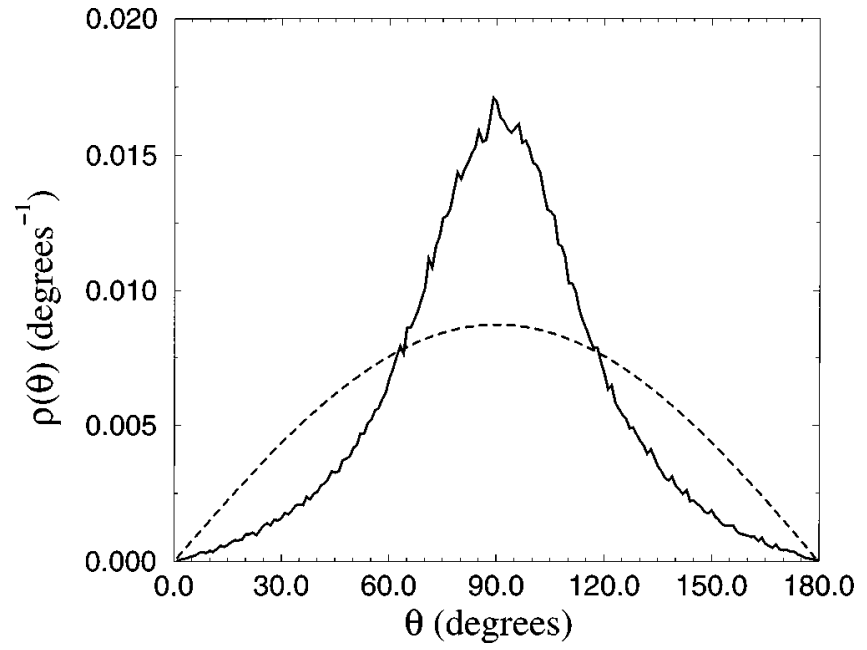

FIG. 4. Normalized orientational distribution of the local fields. $\theta=90^{\circ}$ corresponds to a field in the plane of the benzene ring. The solid line is obtained from the simulation run and the dashed line denotes the distribution corresponding to the case of no directional preference of the local fields.

from its apolar character, we indeed find that benzene is a truly linear substance. Its electrostatics in the absence of an external electric field can be fully quantified in terms of the linear polarizability $\underset{\underline{\alpha}}{\alpha}$. A second feature noted from Fig. 3 is that the nonlinear contributions to the local fields tend to be slightly higher along the molecular $z$ axis.

In Fig. 4, the distribution of angles formed by the local fields $\underline{E}_{\mathrm{loc}, i}$ with the molecular $z$ axis is shown $\left(\theta=0^{0}\right.$ corresponds to alignment of $\underline{E}_{\mathrm{loc}, i}$ with the $z$ axis). The dashed line indicates the distribution that would have been obtained if there had been no directional preference of $\underline{E}_{\mathrm{loc}, i}$. Clearly, the local fields tend to lie in the plane of the molecules (because the molecular polarizability is largest in the plane of the ring).

We conclude from Figs. 2 to 4 that the proposed method is able to capture both the (nonlinear contributions to the) magnitude and the orientational distribution of the local electric fields experienced by the molecules in liquid benzene. We believe this to be of importance in the study of both linear and nonlinear optical material properties: Knowing the strength and orientational distribution of the local field on the molecules may be useful input for ab initio quantum chemical work geared toward quantifying the magnitude of the solvent effect on the (hyper)polarizabilities of polar substances.

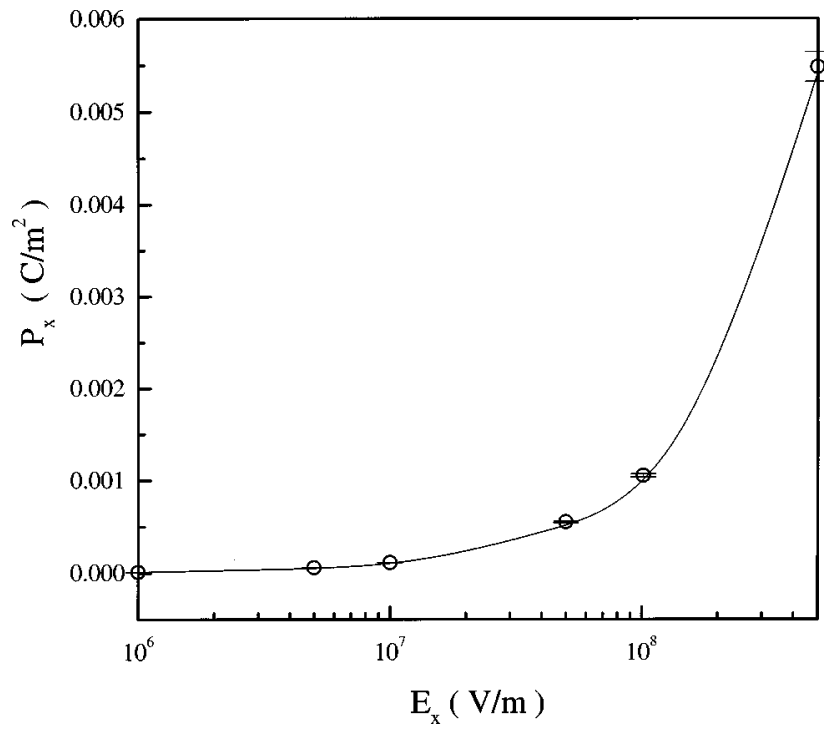

FIG. 5. The points show the dependence of the $X$ component of the macroscopic polarization $P_{X}$ on the macroscopic field $E_{X}$, as obtained by imposing on the model fluid fields of various magnitudes directed along the $X$ axis of the laboratory coordinate frame. The curve is a best fit of the equation $P_{X}=\chi_{X X}^{(1)} E_{X}+\chi_{X X X X}^{(3)} E_{X}^{3}$ to the simulation points. The values of $\chi_{X X}^{(1)}$ and $\chi_{X X X X}^{(3)}$ are extracted from this fit according to the $\underline{P}(\underline{E})$ fit strategy.

\section{Macroscopic optical properties}

Results for the refractive index $n$, obtained from the $\underline{\underline{\chi}}^{(1)}$ values extracted from the simulation according to the three different strategies explained in Secs. II A and IIC, are shown in Table II, in the row labeled "Full model." Some results representative of the performance of the $P(E)$-fit approach are shown in Fig. 5. Table II also lists an experimentally obtained value of the refractive index, ${ }^{52}$ as well as the estimate of $n$ obtained from the commonly employed Lorenz-Lorentz (LL) equation, ${ }^{15}$

$$
\frac{n^{2}-1}{n^{2}+2}=\frac{\rho}{3 \epsilon_{0}} \alpha,
$$

with $\rho$ being the density of the simulated liquid phase and $\alpha$ obtained from the values appearing in Table I as $\alpha$ $=1 / 3 \Sigma_{i} \alpha_{i i}$. The values and error bars for $n$ reported in Table II represent averages and standard deviations of the $n_{I I}$ components monitored along the different axes of the simulation box. In all cases studied, the computed $n$ tensor was found to fulfill the symmetries expected of an isotropic liquid: Offdiagonal elements were equal to zero and diagonal elements were equal to each other within the error of the simulation.

TABLE II. Refractive index $n$.

\begin{tabular}{lccccc}
\hline \hline & $\underline{P}(\underline{E})$ fit & Subtraction & Fluctuation & LL $^{\mathrm{a}}$ & Exptl $^{\mathrm{b}}$ \\
\hline Full model & $1.4912 \pm 0.006$ & $1.4912 \pm 0.0052$ & $1.4918 \pm 0.0095$ & 1.4876 & $\ldots$ \\
Isotropic $\alpha$ & $1.4999 \pm 0.0067$ & $1.4999 \pm 0.0063$ & $1.5005 \pm 0.0063$ & 1.4876 & $\ldots$ \\
& & & & & 1.5011 \\
\hline \hline
\end{tabular}

Value based on the molar density of the simulated fluid, $\rho=11.287 \times 10^{3} \mathrm{~mol} \mathrm{~m}^{-3}$

${ }^{\text {b}}$ Value at $300 \mathrm{~K}, 1 \mathrm{~atm}\left(\rho_{\text {exptl }}=11.237 \times 10^{3} \mathrm{~mol} \mathrm{~m}^{-3}\right)$ for the sodium D line, taken from Ref. 52. 
TABLE III. Second nonlinear susceptibility $\chi^{(3)}, 10^{-33} \mathrm{C}^{4} \mathrm{~m} \mathrm{~J}^{-3}$.

\begin{tabular}{|c|c|c|c|c|c|}
\hline & $\underline{P}(\underline{E})$ fit & Subtraction & Fluctuation & Lorentz & EFISH-expt \\
\hline $\begin{array}{l}\text { Full model } \\
\chi_{I I I}^{(3)} \\
\chi_{\text {IIJJ }}^{(3)}\end{array}$ & $4.55 \pm 0.2$ & $\begin{array}{l}4.577 \pm 0.198 \\
1.520 \pm 0.066^{\mathrm{a}}\end{array}$ & $4.533 \pm 0.196$ & 4.235 & $5.22 \pm 0.79^{\mathrm{b}}$ \\
\hline$\frac{\chi_{I I I I}^{(3)}}{\chi_{I I J J}^{(3)}}$ & & $3.01 \pm 0.05$ & & & $3.03 \pm 0.17^{\mathrm{c}}$ \\
\hline $\begin{array}{l}\text { Isotropic } \underset{\alpha}{\alpha} \chi_{I I I}^{(3)}= \\
\chi_{\text {IIJJ }}^{(3)}\end{array}$ & $5.05 \pm 0.301$ & $\begin{array}{l}5.055 \pm 0.405 \\
1.674 \pm 0.134^{\mathrm{a}}\end{array}$ & $5.008 \pm 0.408$ & & \\
\hline$\frac{\chi_{I I I I}^{(3)}}{\chi_{I I J J}^{(3)}}$ & & $3.02 \pm 0.05$ & & & \\
\hline
\end{tabular}

${ }^{a}$ Averages and error estimates are based on all $\chi_{I I J J}^{(3)}$ elements.

${ }^{b}$ Value obtained from Table I of Ref. 10 by converting the reported macroscopic signal $\Gamma=6.33 \times 10^{-14}$ esu to SI units $\left(1 \mathrm{C}^{4} \mathrm{~m} \mathrm{~J}^{-3}=0.80776 \times 10^{19} \mathrm{esu}\right)$ and using $\chi_{I I I}^{(3)}=2 / 3 \Gamma$, which is valid for EFISH experiments (see Appendix D of Ref. 10 or Ref. 18).

${ }^{\mathrm{c}}$ Value taken from Ref. 47. Measurement is on gaseous benzene.

In Table II we do not show the $\chi^{(1)}$ 's obtained from the run, but these follow immediately from the tabulated refractive indices via Eq. (3).

From Table II it is seen that (a) estimates of $n$ obtained from the three different simulation strategies are in excellent agreement with each other; (b) the LL estimate is within the error bars of the simulated values; (c) both the simulation and the LL approach produce refractive indices that compare well with experiment, although both methods tend to underestimate $n$ slightly. The simulated values tend to be a little higher (and thus closer to experiment) than the LL value, but for liquid benzene the LL approach is clearly preferable as an estimator of $\chi^{(1)}$ and $n$, in view of its simplicity. This may not be so for polar substances, since the local field factor approach underlying Eq. (24) is known to deteriorate with increasing polarity. ${ }^{15}$

A more stringent test of the simulation approach lies in the comparison of the second nonlinear susceptibility $\chi^{(3)}$ to experimentally obtained values. Results obtained by our three simulation strategies are displayed in Table III, section labeled "Full model." Again, estimates from the three simulation strategies agree very well with each other. The isotropic character of liquids dictates that there be only two nonzero elements of $\chi^{(3)}$, i.e., $\chi_{I I I I}^{(3)}$ and $\chi_{I I J J}^{(3)}=\chi_{I J J I}^{(3)}=\chi_{I J I J}^{(3)}$, and that their ratio $\chi_{I I I I}^{(\overline{5})} / \chi_{I I J J}^{(3)}$ be equal to three. ${ }^{9,47,53,55}$ As shown in Table III, our simulation findings are in excellent agreement with this.

Table III also shows a $\chi_{I I I I}^{(3)}$ value obtained from an electric field induced second-harmonic generation (EFISH) experiment reported by Levine and Bethea. ${ }^{10}$ We believe that the value obtained from this experiment is representative of the bulk of the reported values.

From Table III it is seen that the $\chi_{I I I I}^{(3)}$ and $\chi_{I I J J}^{(3)}$ obtained from the simulations compare well with the experimental values. The MD run produces results that are accurate within the error of the simulation and experiment (note that the statistical error in the simulation is much smaller than in the experimental value). These results justify the "a posteriori" electrostatic analysis and confirm the internal consistency of the three strategies adopted here in the case of benzene.

Table III also shows the $\chi_{I I I I}^{(3)}$ 's obtained from the commonly employed Lorentz local field factor equation ${ }^{14}$

$$
\chi_{I I I I}^{(3)}=\rho \gamma f_{o} f_{\omega}^{2} f_{2 \omega}
$$

in which $\rho$ is the density of the simulated liquid phase and the $f$ 's are the so-called local field factors that relate the macroscopic field to the actual local field experienced by the molecules. For $\rho$ we have used the value $11.287 \times 10^{3}$ mol $\mathrm{m}^{-3}$, and for the $f$ 's we have used the standard relations $f_{0}=(\epsilon+2) / 3$ and $f_{\omega}=f_{2 \omega}=\left(n^{2}+2\right) / 3$ with $\epsilon=2.28$ (see Ref. 52) and $n$ equal to the LL estimate listed in the fourth column of Table II. The molecular hyperpolarizability $\gamma$ appearing in Eq. (25) was obtained from the tensor-elements reported in Table I via $\gamma=1 / 15 \Sigma_{i} \Sigma_{j}\left(\gamma_{i i j j}+\gamma_{i j j i}+\gamma_{i j i j}\right)$. This relation corresponds to a Boltzmann averaging over the orientations of a single molecule with respect to a fixed axis (the axis of the dc electric field employed in the EFISH experiments). ${ }^{54}$

Clearly, the Lorentz approach neglects intermolecular correlations that are present and accounted for in the simulation. Furthermore, it assumes molecules to be spherical, instead of ellipsoidal. ${ }^{55}$ The Lorentz approach also neglects correlations between the strength of the local electric field and the molecular position (see pp. 177-180 of Ref. 15), which corresponds to ignoring variations in the strength of the local electric field on molecular length scales. It is therefore not surprising that Eqs. (24) and (25) are in error for many (polar) substances, which is the main reason why we started the development of more quantitative relations between molecular and macroscopic material properties of NLO substances in this work. Lorentz local field-type approaches incorporating the nonsphericity of the benzene molecule have been developed, ${ }^{15,55}$ but, although conceptually superior, they result in optical properties that compare less 
well with experiment for the case of benzene. This, and the fact that the spherical Lorentz local field factors are used by most experimentalists in the NLO field, have led us to only present results obtained from Eqs. (24) and (25) in this paper. Although, as seen in Table III, the uncertainty in the experimental $\chi_{I I I I}^{(3)}$ is large, it is important to note that the simulation approach produces values of $\chi_{I I I I}^{(3)}$ that are by $\sim 8 \%$ higher, and therefore compare more favorably with experiment, than the Lorentz field factor approach using the same molecular $\underline{\underline{\alpha}}, \underline{\underline{\underline{\underline{\gamma}}}}$, and $\rho$ as inputs. In the case of $\underline{\underline{\underline{\chi}}}^{(3)}$, the Lorentz estimate is well outside the error bars of the simulation. In our opinion, in view of the rather crude approximations adopted in Eq. (25), this may well point to an inaccuracy of the local field factor approach. Of course, extensions of our simulation work to more discriminating cases (polar substances) are certainly needed, and are underway.

Two reasons for the observed deviation between simulation predictions for $n$ and $\chi_{I I I I}^{(3)}$ and the corresponding estimates based on $\alpha, \gamma$, and Lorentz local field factors are (a) the anisotropic shape of the fluid molecule, which would necessitate consideration of an ellipsoidal "cavity" of excluded volume in the dielectric continuum representing the fluid around a molecule; (b) the anisotropy of the $\alpha$ tensor of the molecule. In benzene, these two anisotropies partially compensate each other: $\alpha$ elements are higher by almost a factor of 2 within the plane of the benzene ring than normal to the ring, along which direction the molecular center is closest to its surroundings. On the other hand, the polarizable fluid surrounding the disklike excluded volume cavity created by a molecule responds to the field generated by the instantaneous dipole at the center of the molecule more strongly close to the short molecular axis than close to the broad rim of the ring. To assess the effects of the anisotropy of $\underline{\alpha}$ on the linear and nonlinear susceptibilities computed from the simulation, we have repeated all calculations with the same $\underline{\underline{\underline{\gamma}}}$ tensor, but using an isotropic $\alpha=\alpha \underline{\underline{1}}$, with $\alpha$ $=1 / 3 \sum_{i} \alpha_{i i} \underline{\underline{\underline{\underline{\gamma}}}}$. Obtained results are shown in the sections of Tables I and III labelled "Isotropic $\underset{\underline{\alpha} .}{\alpha}$.' Clearly, isotropization of the molecular polarizability tensor results in $\underline{\underline{\chi}}^{(1)}, n$, and $\underline{\underline{\underline{\underline{\chi}}}}^{(3)}$ values which are significantly higher than those obtained using the true $\alpha$ tensor, and farther away from the Lorentz field factor based estimates. This confirms that cavity anisotropy and polarizability anisotropy effects partially compensate each other in the case of benzene.

\section{CONCLUSIONS}

A MD simulation with "a posteriori" electrostatic analysis of the sampled molecular configurations has been used to assess the local fields and the macroscopic linear and nonlinear optical properties of polarizable benzene in the liquid phase. Three different strategies have been invoked, two of them relying on application of external electric fields on the liquid and the third based on analysis of spontaneous fluctuations in the field-free liquid. Results from the three strategies are identical, within simulation error.

The good agreement with experimental data indicates that the static gas-phase (hyper)polarizabilities, calculated from $a b$ initio quantum mechanics and used as input in our simulation work, are sufficient to accurately describe the optical properties of the liquid. Although no solvent effect on the (hyper)polarizabilities was found, we do not expect this to be true in general. Since the approach outlined in this paper is shown to be able to capture the distribution of strengths and orientations of the local fields experienced by the molecules in a liquid phase, we believe that it is ideally suited for combining with $a b$ initio calculations to quantify the magnitude of the solvent effect in polar substances.

In the present contribution, we have also compared estimates of the macroscopic optical properties obtained from the detailed simulation to estimates based on the Lorentz local field factor approach; this is an interesting comparison, as the Lorentz approach is commonly used to extract molecular hyperpolarizabilities from macroscopic signals measured in EFISH experiments. The linear susceptibility and refractive index obtained from the full simulation were found to be practically indistinguishable from the corresponding estimates from the Lorentz approach, the latter being somewhat lower. This good agreement was shown to be due to the fact that molecular shape (cavity) and polarizability anisotropy effects partially compensate each other in the case of benzene; use of an isotropic polarizability tensor in the simulation yields higher values of the linear optical properties, in worse agreement with the Lorentz approach. Simulation estimates of the second nonlinear susceptibility were found to be significantly (by $\sim 8 \%$ ) higher than those based on Lorentz local field estimates; they, too, become even higher if an isotropic molecular polarizability tensor is utilized in the simulation. Further quantifying the accuracy of the local field factor approach in polar or associating substances seems important, since errors associated with this approach may become much larger and thus obscure the assessment of the solvent effect in such substances.

Finally, we believe that there lies ahead an important task for statistical mechanics to help establish quantitative relations between molecular and macroscopic optical properties of NLO materials.

\section{ACKNOWLEDGMENTS}

We gratefully acknowledge support from the European Commission in the form of a TMR Network Grant (Contract No. ERB-FMRX-CT96-0047). We are also indebted to Professor Robert Munn and Dr. Oscar Ahumada for stimulating discussions.

${ }^{1}$ P. N. Prasad and D. J. Williams, Introduction to Nonlinear Optical Effects in Molecules and Polymers (Wiley, New York, 1991).

${ }^{2}$ E. G. J. Staring, Recl. Trav. Chim. Pays-Bas. 110, 492 (1991).

${ }^{3}$ J. L. Bredas, C. Adant, P. Tackx, and A. Persoons, Chem. Rev. 94, 243 (1994).

${ }^{4}$ Nonlinear Optical Properties of Organic Molecules and Crystals, edited by D. S. Chemla and J. Zyss (Academic, New York, 1987).

${ }^{5}$ E. Perrin, P. N. Prasad, P. Mougenot, and M. Dupuis, J. Chem. Phys. 91, 4728 (1989).

${ }^{6}$ C. Daniel and M. Dupuis, Chem. Phys. Lett. 171, 209 (1990).

${ }^{7}$ V. J. Docherty, D. Pugh, and J. O. Morley, J. Chem. Soc., Faraday Trans. 2 81, 1179 (1985).

${ }^{8}$ J. Waite, M. G. Papadopoulos, and C. A. Nicolaides, J. Chem. Phys. 77, 2536 (1982).

${ }^{9}$ J. F. Ward and I. J. Bigio, Phys. Rev. A 11, 60 (1975).

${ }^{10}$ B. F. Levine and C. G. Bethea, J. Chem. Phys. 63, 2666 (1975). 
${ }^{11}$ F. Kajzar, I. Ledoux, and J. Zyss, Phys. Rev. A 36, 2210 (1987).

${ }^{12}$ K. Clays and A. Persoons, Phys. Rev. Lett. 66, 2980 (1991).

${ }^{13}$ I. R. Gentle and G. L. D. Ritchie, J. Phys. Chem. 93, 7740 (1989).

${ }^{14}$ J. A. Armstrong, N. Bloembergen, J. Ducuing, and P. S. Pershan, Phys. Rev. 127, 1918 (1962).

${ }^{15}$ C. J. F. Böttcher, Theory of Electric Polarization (Elsevier, Amsterdam, 1952).

${ }^{16}$ A. Willets and J. E. Rice, J. Chem. Phys. 99, 426 (1993).

${ }^{17}$ J. Yu and M. C. Zerner, J. Chem. Phys. 100, 7487 (1994).

${ }^{18}$ A. Willets, J. E. Rice, D. M. Burland, and D. P. Shelton, J. Chem. Phys. 97, 7590 (1992).

${ }^{19}$ M. Stähelin, C. R. Moylan, D. M. Burland, A. Willets, J. E. Rice, D. P. Shelton, and E. A. Donley, J. Chem. Phys. 98, 5595 (1993).

${ }^{20}$ M. P. Allen and D. J. Tildesley, Computer Simulation of Liquids (Clarendon, Oxford, 1987).

${ }^{21}$ S. W. de Leeuw, J. W. Perram, and E. R. Smith, Proc. R. Soc. London, Ser. A 373, 27 (1980).

${ }^{22}$ D. Frenkel and B. Smit, Understanding Molecular Simulation (Academic, London, 1996).

${ }^{23}$ M. Sprik and M. L. Klein, J. Chem. Phys. 89, 7556 (1988).

${ }^{24}$ G. Ruocco and M. Sampoli, Mol. Phys. 82, 875 (1994).

${ }^{25}$ L. C. Geiger and B. M. Ladanyi, J. Chem. Phys. 87, 191 (1987).

${ }^{26}$ B. M. Ladanyi, J. Chem. Phys. 78, 2189 (1983).

${ }^{27}$ P. A. Franken, A. E. Hill, C. W. Peters, and G. Weinreich, Phys. Rev. Lett. 7, 118 (1961).

${ }^{28}$ R. P. Feynman, The Feynman Lectures on Physics (Addison-Wesley, Reading, MA, 1964), Vol. 2.

${ }^{29}$ R. W. Munn, Mol. Phys. 64, 1 (1988).

${ }^{30}$ M. R. Battaglia, T. I. Cox, and P. A. Madden, Mol. Phys. 37, 1413 (1979).

${ }^{31}$ G. Karlström, P. Linse, A. Wallqvist, and B. Jönsson, J. Am. Chem. Soc. 105, 3777 (1983).

${ }^{32}$ P. Linse, J. Am. Chem. Soc. 106, 5425 (1984).

${ }^{33}$ P. Linse, S. Engström, and B. Jönsson, Chem. Phys. Lett. 115, 95 (1985).

${ }^{34}$ C. J. Craven, P. D. Hatton, and G. S. Pawley, J. Chem. Phys. 98, 8244 (1993).

${ }^{35}$ L. S. Bartell, L. R. Sharkey, and X. Shi, J. Am. Chem. Soc. 110, 7006 (1988).

${ }^{36}$ D. E. Williams and T. L. Starr, Comput. Chem. Eng. 1, 173 (1977).
${ }^{37}$ R. Q. Snurr, A. T. Bell, and D. N. Theodorou, J. Phys. Chem. 97, 13742 (1993).

${ }^{38}$ W. C. Ermler, R. S. Mulliken, and E. Clementi, J. Am. Chem. Soc. 98, 388 (1976).

${ }^{39}$ C. Möller and M. S. Plesset, Phys. Rev. 46, 618 (1934).

${ }^{40}$ J. A. Pople, J. S. Binkley, and R. Seeger, Int. J. Quantum Chem., Quantum Chem. Symp. 10, 1 (1976).

${ }^{41}$ Gaussian 94, M. J. Frisch, G. W. Trucks, H. B. Schlegel, P. M. W. Gill, B. G. Johnson, M. A. Robb, J. R. Cheeseman, T. A. Keith, G. A. Petersson, J. A. Montgomery, K. Raghavachari, M. A. Al-Laham, V. G. Zakrzewski, J. V. Ortiz, J. B. Foresman, J. Cioslowski, B. B. Stefanov, A. Nanayakkara, M. Challacombe, P. Y. Peng, P. Y. Ayala, M. W. Wong, J. L. Andres, E. S. Replogle, R. Gomperts, R. L. Martin, D. J. Fox, J. S. Binkley, D. J. Defrees, J. Baker, J. P. Stewart, M. Head-Gordon, C. Gonzalez, and J. A. Pople, Inc., Pittsburgh PA, 1995.

${ }^{42}$ A. J. Sadlej, Collect. Czech. Chem. Commun. 53, 1995 (1988).

${ }^{43}$ C. Adant, M. Dupuis, and J.-L. Brédas, Int. J. Quantum Chem., Quantum Chem. Symp. 29, 497 (1995).

${ }^{44}$ Numerical Data and Functional Relationships in Science and Technology, edited by K.-H. Hellwege and A. M. Hellwege, Landolt-Börnstein, New Series, Group II: Atomic and Molecular Physics Vol. 7 (Springer, Berlin, 1976).

${ }^{45}$ D. M. Bishop, Rev. Mod. Phys. 62, 343 (1990).

${ }^{46}$ M. P. Bogaard, A. D. Buckingham, M. G. Corfield, D. A. Dunmur, and A. H. White, Chem. Phys. Lett. 12, 558 (1972).

${ }^{47}$ J. F. Ward and D. S. Elliot, J. Chem. Phys. 69, 5438 (1978).

${ }^{48}$ C. J. Craven, P. D. Hatton, C. J. Howard, and G. S. Pawley, J. Chem. Phys. 98, 8236 (1993).

${ }^{49}$ R. Edberg, D. J. Evans, and G. P. Morriss, J. Chem. Phys. 84, 6933 (1986).

${ }^{50}$ G. Ciccotti, M. Ferrario, and J. P. Ryckaert, Mol. Phys. 47, 1253 (1982).

${ }^{51}$ A. H. Narten, J. Chem. Phys. 67, 2102 (1977).

${ }^{52}$ Handbook of Chemistry and Physics, edited by R. C. Weast (CRC, Boca Raton, FL, 1988).

${ }^{53}$ I. J. Bigio and J. F. Ward, Phys. Rev. A 9, 35 (1974).

${ }^{54}$ R. S. Finn, Ph.D. thesis, University of Michigan, 1971

${ }^{55}$ T. Keyes and B. M. Ladanyi, in Advances in Chemical Physics (Wiley, New York, 1984), Vol. LVI, p. 41 